\title{
OCORRÊNCIA DE PARASITOSES INTESTINAIS EM CRIANÇAS NO MUNICÍPIO DE PAU DOS FERROS/RN
}

\section{OCCURRENCE OF INTESTINAL PARASITOSIS IN CHILDREN IN THE CITY OF PAU DOS FERROS/RN}

José Leandro Barbosa Vieira', Márcio Adriano Fernandes Barreto2*, João Bezerra De Queiroz Neto², Antonio Pedro Da Silva Neto ${ }^{3}$, Felipe César Chaves De Oliveira ${ }^{4}$, Ellany Gurgel Cosme do Nascimento'

' Instituto Federal do Rio Grande do Norte/RN, 2Universidade do Estado do Rio Grande do Norte/RN, ${ }^{3 S e c r e t a r i a}$ Municipal de Saúde de Pau dos Ferros/RN, ${ }^{4}$ Secretaria Municipal de Saúde de Riacho de Santana/RN

"Endereço: Universidade do Estado do Rio Grande do Norte-UERN, Campus Avançado Profa $M^{a}$. Elisa de Albuquerque Maia-CAMEAM, Pau dos Ferros, RN, no endereço BR 405, KM 153, Arizona 59900-000 - Pau dos Ferros - RN. Telefone: (84) 3351-2560, e-mail: marciobioquimico@hotmail.com

\section{RESUMO}

O estudo analisa a ocorrência de parasitoses intestinais em crianças matriculadas na rede municipal de educação infantil em Pau dos Ferros - RN e identifica quais fatores sócios demográficos que influenciam. Através da análise parasitológica das fezes, pelo método de Hoffman em 128 crianças e aplicação de questionário socioeconômico com os pais ou responsáveis, a seleção ocorreu através de amostragem aleatória simples. A ocorrência de parasitos intestinais foi evidenciada em 59,4\%, destes $97,4 \%$ infectadas com protozoários e 2,6\% com helmintos. Os protozoários mais prevalentes foram: Endolimax nana e Giardia lamblia, 0 único helminto detectado foi o Enterobius vermicularis, com 1,6\%. O poliparasitismo ocorreu em $9,4 \%$ dos casos. Observou-se uma frequência mais elevada em crianças residentes em ruas não pavimentadas; com renda familiar inferior a 1 salário mínimo; pertencentes às famílias com 6 a 8 membros no domicílio; que se utilizam do sistema de fossa para o destino dos dejetos e que consumiam água sem tratamento domiciliar. Conclui-se a necessidade de investimentos em infraestrutura básica e políticas públicas voltadas para a promoção de um ambiente salutar e de educação em saúde, podendo contribuir para uma redução significativa na prevalência de parasitos intestinais nas crianças em idade pré-escolar.

Palavras-Chave: Parasitoses intestinais. Ocorrência. Escolares. Fatores socioeconômicos. Pau dos Ferros/RN

\section{ABSTRACT}

This study aimed at analyzing the occurrence of intestinal parasites in children enrolled in the municipal elementary schools system in the city of Pau dos Ferros/RN. It was identified parasitological analysis of feces, using Hoffman's method in 128 children enrolled in the public daycare centers, and we also did it through the application of socioeconomic questionnaires to their parents or their legal guardian. The selection occurred through simple random sampling. The occurrence of intestinal parasites was at a rate $59,4 \%$ of children. $97.4 \%$ of those children were infected with protozoa and $2.6 \%$ with helminths. The most prevalent protozoa were Endolimax nana, and Giardia lamblia. The only helminth detected was the Enterobius vermicularis, $1.6 \%$ of prevalence. The polyparasitism occurred in $9,4 \%$ of the cases. It was observed a correlation between the 
occurrence of intestinal parasites and children resident in not paved streets; with a familiar income inferior than 1 minimum wage, those children that are part of families from 6 to 8 members living at the same house. It is necessary to say that they use the same cesspit system for the destination of waste and that they consume water without the necessary treatment. It can be concluded that investments in basic infrastructure and also that public politics directed to the promotion of a healthy environment and education in health can contribute for a significant reduction in the prevalence of intestinal parasites in children in pre-school age in the city of Pau dos Ferros, RN. Keywords: Parasitic illnesses. Occurrence. School. Socioeconomics factors. Pau dos Ferros/RN

\section{INTRODUÇÃO}

Considerando que As parasitoses intestinais configuram-se através dos seus veículos de transmissão (a água e os alimentos), esta temática tem se tornadouma preocupação a ser discutida em nosso país, uma vez que vivenciamos uma carência de infraestrutura sanitária. Paralela a essa realidade, podem ser citados a baixa escolarização e a ingestão de alimentos com precários hábitos de higiene pessoal como fatores que acentuam ainda mais a disseminação das parasitoses intestinais. Dessa forma, esse conjunto de elementos adquire muita força em espaços onde não se tem um controle da qualidade da água e dos alimentos, como em escolas, creches, presídios e/ou outros espaços coletivos ${ }^{1}$.

Nesse contexto, de doenças infecciosas e parasitárias, tem-se uma das principais causas de morte em todo o mundo e que, desafortunadamente, continua a figurar no âmbito mundial. Logo, alguns fatores são condições propícias para multiplicação do parasito junto a uma população suscetível, como as alterações ambientais, a elevada concentração populacional e a falta de higiene ${ }^{3}$. No Brasil esse problema é agravado justamente devido às precárias condições de saneamento básico, ao baixo nível socioeconômico, à falta de orientação sanitária e de programas de educação para a saúde. Estimativas referentes à frequência de enteroparasitoses na infância relataram prevalências variando de $10,7 \%$ a $89 \%$, conforme a região e o período estudados ${ }^{2}$.

Paralelo a essa questão as crianças são as mais vulneráveis,em decorrência de fatores inerentes à pouca idade, ao desenvolvimento imunológico incompleto, aos hábitos de higiene deficientes e também devido a um maior contato com o solo no cotidiano de suas brincadeiras. Assim, percebe-se que na faixa etária de 3 a 6 anos são encontradas as maiores frequências de infecção e diagnóstico de casos. Já na faixa de 6 a 9 anos, apesar dessa incidência continuar elevada, existe uma tendência ao decréscimo mediante o aumento da faixa etária4.

Nessa perspectiva, níveis elevados de mono e poliparasitismo, associados à alimentação com baixos níveis de nutrientes, podem comprometer o desenvolvimento adequado do organismo humano, sobretudo nos primeiros anos de vida. Como consequência, esses fatores desencadeiam implicações econômicas, pois o aumento de doenças implica em aumento do uso da assistência, isto é, aumento dos gastos familiares com cuidados médicos e medicamentos ${ }^{8}$, além de problemas gastrintestinais, baixo rendimento corporal e, por consequência, atraso no desenvolvimento escolar, se constituindo, nesse sentido, em um problema de ordem sanitária e social ${ }^{2,5}$.

Em decorrência da urbanização, assim como da crescente participação feminina no mercado de trabalho, as creches passaram a ser o primeiro ambiente externo (em relação ao doméstico) que 
a criança frequenta, tornando-se lugares potenciais de contaminação ${ }^{6}$. Além disso, as crianças que frequentam creches têm maior contato entre si, favorecendo o processo de transmissão pessoa-a-pessoa de helmintos e de protozoários, principalmente Giardia lamblia?.

Diante de tal realidade e, tendo em vista que são muitos os pequenos municípios brasileiros com características sociodemográficas semelhantes, principalmente na região Nordeste, procurou-se estabelecer a ocorrência de parasitoses intestinais em crianças matriculadas na rede municipal de educação infantil da cidade de Pau dos Ferros - RN e identificar quais fatores sociodemográficos influenciam a infecção das parasitoses intestinais.

\section{MATERIAL E MÉTODO}

Este estudo é de caráter transversal, de cunho quantitativo e abordagem descritiva. A pesquisa expõe dados e tendências observáveis sobre a ocorrência das parasitoses intestinais entre os pré-escolares da rede pública do município de Pau dos Ferros - RN.

O local do estudo foi nas creches do município de Pau dos Ferros, que pertence a mesorregião do Alto Oeste Potiguar, tendo uma população de 27.745 habitantes, sendo que 25.551 destes residem na zona urbana e apenas 2.194 na zona rural, que totaliza em questões territoriais em uma área de $259,98 \mathrm{~km}^{2}$ a referida cidade.A região em que o município se encontra apresenta um clima semiárido e temperaturas elevadas ${ }^{9}$.

Os participantes desta pesquisa foram os alunos matriculados na educação infantil, bem como os pais ou responsáveis dessas crianças, no município de Pau dos Ferros - RN. Essas crianças totalizam-se em uma população de 696 com idade entre 3 a 6 anos de idade, matriculados nas creches do município.

Entretanto, a amostra obtida, no término da coleta dos dados, foi de apenas128 alunos, em virtude dos critérios de inclusão, quais sejam: estar devidamente matriculado na escola, aceitar participar voluntariamente, no momento da coleta e participar das duas técnicas de coleta de dados. A amostra resultou em 128 alunos com exames parasitológicos realizados e questionários respondidos pelos pais ou responsáveis das crianças.

As creches que foram espaços da pesquisa se localizam nos Bairros "Manoel Domingos" (creche 01), "Riacho do Meio" (creche 02), "Manoel Deodato" (creche 03) e "Paraíso" (creches 04 e 05). Todos estes bairros têm problemas de infraestrutura, com ruas não pavimentadas e esgotos a céu aberto, sendo uns mais acentuados que outros em relação ao nível socioeconômico. Podemos citar como mais carentes os bairros "Manoel Deodato" e "Riacho do Meio". Já o Bairro São Judas Tadeu demonstra uma condição infraestrutural melhor que os bairros anteriormente citados (creche 05), enquanto a zona rural "Perímetro Irrigado" (creche 06) apresenta problemas infraestruturais mais corriqueiros, como falta de saneamento e famílias de condições socioeconômicas menos favorecidas. A coleta transcorreu no período de junho a setembro de 2012, mediante a aplicação de questionário socioeconômico e coleta de amostras fecais, objetivando a realização do Exame Parasitológico de Fezes (EPF).

As crianças foram selecionadas por amostragem aleatória simples. Já os coletores de amostras fecais e os questionários foram numerados para posterior cruzamento dos dados (Ex. coletor 1 questionário 1). Os pais ou responsáveis assinaram o Termo de Consentimento Livre e Esclarecido - TCLE e se prontificaram a devolver o questionário devidamente respondido e as amostras de fezes 
no dia posterior no mesmo espaço da creche onde foi realizado o sorteio das crianças e a entrega dos coletores e questionários.

Foram definidos os seguintes critérios de inclusão: estar devidamente matriculado na escola selecionada, aceitar participar voluntariamente e participar das duas técnicas de coleta de dados. Como critérios de exclusão foram definidos não estar em sala de aula no momento da coleta dos dados e o pai ou responsável legal se negar a autorizar a participação da criança.

As amostras fecais foram transportadas em uma caixa térmica, identificadas apenas com o número correspondente à criança selecionada. Essas amostras foram levadas diretamente da creche para a preparação e análise no laboratório de Morfologia do Curso de Enfermagem do Campus

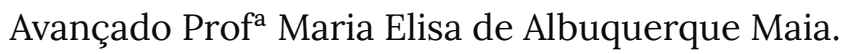

Para a posterior entrega dos resultados dos exames parasitológicos de fezes aos responsáveis das crianças foi necessário armazenar os nomes completos, sexo e idade das crianças selecionadas. Assim, os resultados dos EPF foram entregues aos responsáveis legais pelas crianças, impressos e lacrados, orientando-os também para buscar atendimento com o médico da Estratégia de Saúde da Família de sua comunidade.

As análises parasitológicas das fezes foram executadas por método de Hoffman, Pons e Janer modificado. Quanto aos resultados dos estudos, estes foram tabulados no programa SPSS versão 20 e a análise foi trabalhada por estatística simples e bivariada, do tipo qui-quadrado, com significância estatística quando $\mathrm{p}<0,05$.

O presente estudo atendeu às diretrizes e normas da Resolução n ${ }^{\circ}$ 196/96 do Conselho Nacional de Saúde e foi aprovado pelo Comitê de Ética em Pesquisa (CEP) da Universidade do Estado do Rio Grande do Norte (UERN) com a CAAE nº 0149.0.428.000-11 em 04 de maio de 2012.

\section{RESULTADOS}

Foram entrevistados 128 responsáveis pelas crianças e realizados 128 exames parasitológicos das crianças. Levando em consideração que a média de pessoas por domicílio foi 4,03, esta pesquisa abrange a realidade de 512 sujeitos. Dentre os entrevistados, 57\% eram do sexo feminino e 89,8\% destes residem na zona urbana. A média de idade foi de 4,18 anos. Em relação ao tipo de moradia, 96,9\%, reside em casas de alvenaria, 36,7\% vivem com lixo próximo as suas casas.

Tabela 1 - Perfil socioeconômico e a ocorrência de parasitoses intestinais entre os pré-escolares das creches de Pau dos Ferros - RN, 2012.

\begin{tabular}{l|l|l} 
VARIÁVEL & Participantes & $\begin{array}{l}\text { POSITIVOS } \\
\mathbf{N}^{\circ} \mathbf{( \% )}\end{array}$ \\
\hline Escolaridade da Mãe & & $25(60,9)$ \\
\hline Fundamental Incompleto & 41 & $10(58,8)$ \\
\hline Fundamental Completo & 17 & $11(74,4)$ \\
\hline Médio Incompleto & 15 & $20(47,6)$ \\
\hline Médio Completo & 42 & $0(0,0)$ \\
\hline Superior Incompleto & 1 & $1(50)$ \\
\hline Superior Completo & 2 & $8(80)$ \\
\hline Não Informado & 10 &
\end{tabular}




\begin{tabular}{l|l|l}
$\begin{array}{l}\text { VARIÁVEL } \\
\text { Renda Familiar: }\end{array}$ & Participantes & $\begin{array}{l}\text { POSITIVOS } \\
\mathbf{N}^{\circ}(\mathbf{\%})\end{array}$ \\
\hline $\mathrm{R} \$ 100,00$ a R 550,00 & 68 & $44(64,7)$ \\
\hline $\mathrm{R} \$ 551,00$ ou mais & 58 & $32(55,2)$ \\
\hline Não Informado & 2 & $0(0,0)$ \\
\hline Água consumida: & & \\
\hline Sem Tratamento & 15 & $12(80)$ \\
\hline Mineral & 65 & $36(55,4)$ \\
\hline Filtrada & 46 & $27(58,7)$ \\
\hline Fervida & 2 & $1(50)$ \\
\hline Rua de Moradia: & & \\
\hline Não Pavimentada & 74 & $48(64,9)$ \\
\hline Pavimentada & 53 & $28(52,8)$ \\
\hline Não Informado & 1 & $0(0,0)$ \\
\hline Destino dos Dejetos: & & \\
\hline A Céu Aberto & 2 & $1(50)$ \\
\hline Fossa & 97 & $61(62,9)$ \\
\hline Esgoto & 28 & $14(50)$ \\
\hline Não Informado & 1 & $0(0,0)$ \\
\hline & &
\end{tabular}

Quanto ao número de cômodos dos domicílios e a quantidade de pessoas na residência, as médias foram $4,84( \pm 1,48)$ e 4,04 $( \pm 1,12)$. Das crianças participantes do estudo $88,3 \%$ lavam as mãos após usarem o banheiro e 85,9\% lavam as mãos antes das refeições e 38,3\% tinham o hábito de andar descalço.

Analisando se houve infecções anteriores por parasitas intestinais nos escolares e em seus familiares, 57,8\% responderam que já havia apresentado algum tipo de parasita intestinal. Dos familiares que residem junto com os participantes da pesquisa, $59,1 \%$ tinham apresentado algum parasita intestinal.

Dessa forma, os exames Parasitológicos de Fezes (EPF) revelaram 76 crianças infectadas perfazendo 59,4\% contendo ao menos um tipo de parasito ou comensal intestinal. Dentre as crianças parasitadas, o percentual de parasitoses por protozoários foi de 97,4 \%, enquanto a ocorrência de helmintos em apenas 2,6\%. As tabelas 2 e 3 estão apresentadas considerando somente as crianças parasitadas, considerando somente $76(59,4 \%)$ da amostra.

Tabela 2 - Frequência de parasitas e comensais intestinais em pré-escolares residentes em Pau dos Ferros - RN, 2012.

\begin{tabular}{l|c|c}
\hline Espécie & Frequência & (\%) \\
\hline Endolimax nana & 25 & 19,5 \\
\hline Entamoeba coli & 9 & 7,0 \\
\hline E. histolytica & 6 & 4,7 \\
\hline Giardia lamblia & 22 & 17,2 \\
\hline Enterobius vermicularis & 2 & 1,6 \\
\hline Poliparasitoses & 12 & 9,4 \\
\hline
\end{tabular}


A ocorrência foi maior (68,3\%) em crianças de famílias com 6 a 8 pessoas na residência, enquanto nas crianças cujas famílias possuíam entre 2 a 5 componentes, a ocorrência foi de 55,2\%.

Tabela 3 - Frequência de parasitoses intestinais associados a idade dos pré-escolares e o grau de escolaridade das mães ou responsáveis, das creches de Pau dos Ferros - RN, 2012.

\begin{tabular}{c|c|c}
\multicolumn{1}{c|}{ Variáveis } & Frequência & $\%$ \\
\hline Idade dos Sujeitos & 4 & $5,2 \%$ \\
\hline 2 & 17 & $22,3 \%$ \\
\hline 3 & 23 & $30,2 \%$ \\
\hline 4 & 20 & $26,3 \%$ \\
\hline 5 & 12 & $15,7 \%$ \\
\hline 6 & 35 & $60,3 \%$ \\
\hline Nível de Escolaridade & 33 & $55 \%$ \\
\hline 9 a 8 Anos de Estudo 16 Anos de Estudo & 8 & $80 \%$ \\
\hline
\end{tabular}

O hábito de andar descalço é bastante comum entre os infantes e tal fato pode favorecer a transmissão de alguns helmintos pela penetração de larvas através da pele. Na pesquisa, este fator não se mostrou relevante para a ocorrência de parasitos. A maior frequência encontrada $(65,5 \%)$ foi no grupo de crianças que não tinham o hábito de andar descalço.

\section{DISCUSSÃO}

A frequência geral obtida foi elevada e se assemelha a estudos realizados em outros estados com prevalência de $58,8 \%{ }^{11}$ e $60,8 \%{ }^{15}$ assim como se assemelha a prevalência superior de protozoários em relação aos helmintos ${ }^{13}$, fato atribuído às características de transmissão de grande parte destes organismos, que está relacionada à penetração de larvas através da pele. Larvas estas que só se tornam viáveis em condições favoráveis de umidade do solo e temperatura ambiental $\left(25\right.$ a $\left.30^{\circ} \mathrm{C}\right)$. Com relação à baixa prevalência de Geohelmintos nestes estudos, pode-se inferir que a disseminação das helmintíases na região nordeste do país está em estreita dependência com a umidade do solo. Dado que, considera-se que nas regiões semiáridas, a longa estação seca é uma das circunstâncias limitantes para a proliferação destes parasitos ${ }^{12,14,15}$. Outro fator que pode ter influenciado esta baixa frequência foi o método de análise parasitológica utilizado, tendo em vista que muitos helmintos, inclusive o próprio E. vermicuralis, ou o S. stercoralis, têm métodos de análise específicos, sendo de difícil detecção pelo método de sedimentação espontânea ${ }^{2,16}$.

Esta baixa prevalência de Geohelmintos pode justificar o fato da menor ocorrência de infecção (44,5\%) entre as crianças participantes do estudo, que tinham o hábito de andar descalço. Em decorrência da grande prevalência de protozoários $(97,4 \%)$ que têm transmissão fecal oral, em relação aos helmintos $(2,6 \%)$, com estratégia de infecção a penetração transcutânea de larvas, o fato de andar ou não descalço não teria influência real na prevalência encontrada.

A grande ocorrência de E. nana, E. coli, apesar de não ter grande relevância clínica, representa grande importância epidemiológica, pois indica contaminação fecal da água e/ou dos alimentos ingeridos por estes infantes ${ }^{2,17,16}$, seja proveniente de suas residências, ou mesmo de seus respectivos estabelecimentos de ensino. Dessa forma, essa questão reflete as condições de saneamento básico, 
a presença ou não de rede de esgoto, a qualidade da água consumida e os hábitos de higiene à que os escolares estão expostos ${ }^{18}$.

Com isso, observou-se maior ocorrência de enteroparasitoses nas faixas etárias com 3 a 5 anos, devido a, possivelmente,já manifestarem uma maior autonomia em suas atividades, interagirem de maneira mais intensa com outras crianças, entrar em nas suas brincadeiras em maior contato com o solo e já utilizarem o banheiro sem supervisão direta dos pais, favorecendo assim a uma higiene deficiente feita por elas mesmas que propicia à transmissão de parasitos intestinais.

Em contrapartida, a menor ocorrência observada foi na faixa etária de dois anos de idade, pois crianças dessa idade ainda não têm tanta autonomia e não realizam as mesmas atividades das crianças mais velhas sem a supervisão dos pais, tornando-se um fator que pode ser importante para o controle das enteroparasitoses. Quanto à maior ocorrência de parasitos no grupo de crianças que utiliza o sistema de fossa como destino final dos dejetos, o resultado vai de encontro a pesquisa de Vasconcelos et al (2011) ${ }^{11}$ que revelou uma predominância de utilização de fossas sépticas entre as crianças parasitadas por protozoários.

A renda familiar sempre foi visualizada como um elemento importante na inserção social das pessoas, influenciando diretamente na morbidade dos sujeitos já que o acesso a bens de consumo e a serviços como saúde e educação são determinantes direta ou indiretamente pela renda dos indivíduos/famílias.

Apesar de não ter apresentado significância estatística nos nossos achados,o estudo realizado aponta que 75,7\% das famílias de crianças parasitadas viviam com, no máximo, um salário mínimo de renda ${ }^{19}$. Consoante a isso, alguns estudos já comprovam a relação linear entre infecção por helmintos e protozoários e as variáveis socioeconômicas. Assim, as crianças que pertencem à famílias de baixa renda, apresentaram maior frequência de infecção parasitária ${ }^{13}$. Portanto, ficou demonstrado também que a renda familiar consiste em fator de proteção para as parasitoses intestinais, observando-se o decrescente número de parasitoses intestinais à medida que a renda familiar aumenta ${ }^{14}$. A maior ocorrência entre as crianças com maior número de familiares residentes no mesmo domicílio indica um favorecimento à transmissão de parasitos, pois estudos mostram que em famílias com mais de uma pessoa por cômodo, aliada a renda de menos de um salário mínimo, possui riscos de transmissão mais elevados ${ }^{8,16}$.

A maior ocorrência entre as crianças de mães com menos anos de estudo é reflexo do nível socioeconômico e cultural. Assim, em virtude dessa questão influenciar nas condições de higiene pessoal e cuidados com a água e os alimentos, pode-se inferir que em classes menos favorecidas estes cuidados não são rigorosamente observados ${ }^{9}$. Dessa forma, fica evidente que as mães com maior escolaridade tiveram mais acesso à informações sobre desenvolvimento infantil, interagem melhor com seus filhos, respondem adequadamente às suas solicitações e podem prover melhores condições físicas e emocionais para o seu desenvolvimento ${ }^{3}$.

A alta frequência entre as crianças que consumiam água sem tratamento domiciliar vai de encontro as pesquisas de Oliveira et al $(2010)^{20}$, que observaram uma prevalência de $66 \%$ entre os participantes que não adicionavam nenhum tratamento domiciliar à água consumida. Em contrapartida, Belo et al (2012) $)^{21}$ constataram em sua pesquisa que o uso do filtro de água nas residências mostrou-se fortemente associado à redução da prevalência geral de helmintos e protozoários. O mesmo não ocorreu com relação ao tratamento realizado em redes gerais, sugerindo assim a necessidade de os próprios moradores realizarem tratamento adicional à água. 
Os hábitos de higiene, principalmente a lavagem das mãos, se mostram como uma estratégia eficaz no controle da transmissão das doenças parasitárias, tendo em vista que a transmissão interpessoal e a reinfecção podem acontecer através do contato com mãos contaminadas com cistos de protozoários ou ovos de helmintos, reiniciando assim um novo ciclo da doença no organismo. Entretanto, na realidade da pesquisa estes hábitos, isoladamente, adotados por parte das crianças não se mostraram eficientes na diminuição da frequência detectada.

Desse modo, a ocorrência elevada em crianças que não andam descalços pode ser explicada em decorrência da baixa frequência de helmintos, tendo em vista que alguns deles têm como forma de transmissão a penetração de larvas através da pele e mucosas, enquanto os protozoários, que foram mais prevalentes na pesquisa, têm sua transmissão favorecida através da ingestão de água e alimentos contaminados com cistos. Assim, o fato de andar ou não descalço não trouxe influência real na prevalência em nosso grupo avaliado.

Um grande número de crianças do estudo mostrou-se infectadas, mesmo após realização do tratamento antiparasitário, que é simples e eficaz. Entretanto, um ponto crucial é a reinfecção dos sujeitos, situação corroborada pelos achados de que há associação entre familiares já infectados com parasitos intestinais e EPF positivo. Assim, infere-se, conforme evidenciado em diversos estudos, que os fatores socioeconômicos, como acesso à saúde e educação, abastecimento de água, renda, saneamento básico, entre outros aspectos, interferem diretamente na cadeia de transmissão dos parasitos, tornando assim um ciclo difícil de ser interrompido apenas com a ação curativa individual ${ }^{3,20,16,21}$.

\section{CONSIDERAÇÕES FINAIS}

A partir do nosso estudo, conseguiu-se observar uma ocorrência geral elevada que superou a hipótese inicial de $50 \%$ de prevalência. A frequência de protozoários foi superior a de helmintos, que já era esperado devido às características climáticas da região.

Houve uma grande ocorrência de organismos comensais, tal fato revela uma contaminação da água consumida pela população estudada, revelando assim uma importância epidemiológica e a necessidade de intervenções no controle de qualidade da água ofertada a população do município.

Conforme demonstrado, grande parte das crianças estavam reinfectadas por parasitos, já que haviam apresentado um quadro de parasitoses intestinais previamente tratados com antiparasitários, demonstrando assim que o tratamento clínico individual não é suficiente para a interrupção do ciclo destes patógenos, mostrando a importância dos determinantes socioeconômicos, culturais e ambientais nesta problemática.

Portanto, infere-seque, devido possivelmente, a realidade dos municípios da região ser semelhante ao encontrado neste estudo, revela-se assim a necessidade de adoção de políticas públicas voltadas para a promoção de um ambiente salutar, de melhorias socioeconômicas, educação sanitária e em saúde, objetivando a promoção de hábitos saudáveis entre a população. Nessa medida, é de extrema importância a interrupção da cadeia de transmissão destas doenças que prejudicam o desenvolvimento das crianças, principalmente àquelas das camadas mais pobres que, convivendo com a miséria e o abandono, ainda tem de conviver com este problema típico do subdesenvolvimento.

No nosso estudo, encontramos algumas limitações como dados que não apresentaram significância estatística durante o cálculo de qui-quadrado que demonstrasse diferença nos achados, possivelmente pelo tamanho da amostra. No entanto, ressalta-se que convivemos em uma região 
com inexistência em quase toda sua totalidade de saneamento básico, tampouco existe um sistema de drenagem onde ocorra o tratamento da água ou do lixo, inclusive com muitas áreas de esgoto a céu aberto. Assim, esses fatores contribuem para facilitar uma contaminação com parasitas independente do perfil sociodemográfico da cidade.

Outro fator limitante foi o método de análise laboratorial utilizado, que não é considerado o padrão ouro nas pesquisas, podendo ter resultado em limitação de diagnóstico e de análise estatística. Entretanto mediante a indisponibilidade de financiamento, este era o método de mais fácil acesso devido ao menor custo e maior facilidade no preparo e análise, além de ainda ser muito utilizado em várias pesquisas.

\section{AGRADECIMENTOS}

Nosso reconhecimento e gratidão a todos as crianças e pais/responsáveis que aceitaram participar do presente estudo, assim como os profissionais das creches. Agradecemos ainda ao professor de Inglês da Universidade do Estado do Rio Grande do Norte (UERN). O trabalho teve o apoio do projeto de extensão PROEPA (Projeto de extensão em Parasitologia) da Universidade do Estado do Rio Grande do Norte (UERN).

\section{REFERÊNCIAS}

1 - Antonio PSN, Nathália LDS, Francisco GV, João BQN, Márcio AFB. Ação e prevenção: uma avaliação parasitológica em manipuladores de alimentos e escolares.Revista Extendere 2013, 1 (2): 23-35.

2 - Saturnino, ARD; Nunes, JFL; Silva, EMA. Relação entre a ocorrência de parasitos intestinais e sintomatologia observada em crianças de umacomunidade carente de Cidade Nova, em Natal Rio Grande do Norte, Brasil. Rev Brasileira de Analises Clinicas 2003; 35 (2) 85-86.

3 - Ferreira, H, Lala, ERP; Monteiro, MC; Raimondo, ML. Estudo epidemiológico localizado da freqüência e fatores de risco para enteroparasitoses e sua correlação com o estado nutricional de crianças em idade pré-escolar parasitoses intestinais e desenvolvimento infantil. Publ. UEPG Biol. Health Sci. 2006; 12 (4): 33-40.

4 - Ludwig, KM; Frei, F; Filho, FA; Paes, JTR. Correlação entre condições de saneamento básico e parasitoses intestinais na população de Assis, Estado de São Paulo. Revista da Sociedade Brasileira de Medicina Tropical 1999; 32 (5): 547-555.

5 - Silva, EF; Silva, VBC; Freitas, FLC. Parasitoses intestinais em crianças residentes na comunidade ribeirinha são Francisco do Laranjal, município do Coari, Estado do Amazonas, Brasil. Revista de Patologia Tropical 2012; 41(1): 97-101.

6 - Monteiro, AMC; Silva, EF; Almeida, KS; Sousa, JJN; Mathias, LA; Baptista, F; Freitas, FLC. Parasitoses intestinais em crianças de creches públicas localizadas em bairros periféricos do município de Coari, Amazonas, Brasil. Revista de Patologia Tropical 2009; 38(4): 284-290.

7 - Machado, RC; Marcari, EL; Cristante, SFV; Carareto, CMA. Giardíase e helmintíases em crianças de creches e escolas de $1^{\circ}$ e $2^{\circ}$ graus (públicas e privadas) da cidade de Mirassol (SP, Brasil).

Revista da Sociedade Brasileira de Medicina Tropical 1999; 32 (6) 697-704.

8 - Nesti, M. M. M; Goldbaum, M. Infectious diseases and daycare and preschool education. Jornal de Pediatria, Rio de Janeiro 2007; 83 (4): 299-312. 
9- IBGE. Instituto Brasileiro de Geografia e Estatística. Rio Grande do Norte - Pau dos Ferros. Censo 2010; [acessado em 03 Abr 2014]. Disponível em: http://www.cidades.ibge.gov.br/xtras/perfil.php?! ang $=\&$ codmun=240940\&search=rio-grande-do-norte\%7cpau-dos-ferros.

10 - Maia, MMM; Fausto, MA; Vieira, ELM; Benetton, MLFN; Carneiro, M. The prevalence of malnutrition and its risk factors in children attending outpatient clinics in the city of Manaus, Amazonas, Brazil. Archivos Latino Americanos de Nutricion 2008; 58 (3): 234-240.

11- Vasconcelos, IAB; Oliveira, JW; Cabral, FRF; Coutinho, HDM; Menezes, IRA. Prevalência de parasitoses intestinais entre crianças de 4-12 anos no Crato, Estado do Ceará: um problema recorrente de saúde pública. Acta Scientiarum. Health Sciences 201 1; 33 (1): 35-41.

12 - Alves, JR; Macedo, HW; Ramos Jr, NA; Ferreira, LF; Gonçalves, ML, C, Araújo, A. Parasitoses intestinais em região semi-árida do nordeste do Brasil: resultados preliminares distintos das prevalências esperadas. Cadernos de Saúde Pública 2003; 19 (2): 667-670.

13 - Carvalho, OS; Guerra, HL; Campos YR; Caldeira RL; Massara, CL. Prevalência de helmintos intestinais e três mesorregiões do estado de Minas Gerais. Revista da Sociedade Brasileira de Medicina Tropica 2002; 35 (6): 601-607.

14 - Silva, FS; Paulo, ADC; Braga, CMM; Almeida, RJ; Galvão, VP. Frequência de parasitos intestinais no município de Chapadinha, Maranhão, Brasil. Revista de patologia Tropical 2010; 39 (1): 63-68.

15 - Vinha, C. Incidência de ancilostomídeosascaris e trichiuris no Brasil. Rev. Bras. de Malariologia e Doenças Tropicais 1969; $21: 549-557$

16 - Visser, S, Giatti, LL, Carvalho, RAC, Guerreiro, JCH. Estudo da associação entre fatores socioambientais e prevalência de parasitoses intestinais em área periférica da cidade de Manaus. Ciência e Saúde Coletiva 2011; 16 (8): 3481-3492.

17 - Devera, R; Requena, I; Blanco, Y; Rumhein, FA; Velasquuez, V; Tedesco, RM. Prevalencia de parasitos intestinales en escolares de la escuela básica estadal José Félix Blanco, estado Bolívar, Venezuela. Salus Online 2010; 14 (3): 43-52.

18 - Seixas, MTL; Souza, JN; Souza, RP; Teixeira, MCA; Soares, NM. Avaliação da frequência de parasitos intestinais e do estado nutricional em escolares de uma área periurbana de Salvador, Bahia, Brasil. Revista de Patologia Tropical 201 1; 40 (4): 304-314.

19 - Teixeira, JC; Heller, L. Fatores ambientais associados às helmintoses intestinais em áreas de assentamento subnormal, Juiz de Fora, MG. Engenharia Sanitária e Ambiental2004; 9 (4): 301-305.

20 - Oliveira, LM; Ferreira, WA; Vasquez, FG; Barbosa, MGV. Parasitoses intestinais e fatores socioambientais de uma população da área periurbana de Manaus - AM. RBPS, 2010; 23 (4): $307-$ 315

21 - Belo, VS; Oliveira, RB; Fernandes, PC; Nascimento, BWL; Fernandes, FV; Castro, CLF; Santos, WB; Silva, ES. Fatores associados à ocorrência de parasitoses intestinais em uma população de crianças e adolescentes. Revista Paulista de Pediatria 2012; 30 (2): 195-201. 